\title{
Carnets
}

Revue électronique d'études françaises de l'APEF

Deuxième série - $12 \mid 2018$

Théorie Mimétique et Études Littéraires

\section{La mise en scène de l'écrivain comme sacrifié dans la littérature du premier romantisme allemand : une nouvelle forme de sacrifice?}

Novalis et la mise en scène de l'écrivain à la lumière des théories de René Girard

\section{Helene Tessier Amorim}

\section{(2) OpenEdition}

Journals

Édition électronique

URL : http://journals.openedition.org/carnets/2493

DOI : $10.4000 /$ carnets.2493

ISSN : 1646-7698

Éditeur

APEF

Référence électronique

Helene Tessier Amorim, «La mise en scène de l'écrivain comme sacrifié dans la littérature du premier romantisme allemand : une nouvelle forme de sacrifice? ", Carnets [En ligne], Deuxième série -

12 | 2018, mis en ligne le 31 janvier 2018, consulté le 05 mai 2019. URL : http://

journals.openedition.org/carnets/2493; DOI : 10.4000/carnets.2493

Ce document a été généré automatiquement le 5 mai 2019.

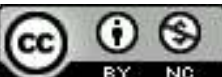

Carnets est mis à disposition selon les termes de la licence Creative Commons - Atribution - Pas d'utilisation commerciale 4.0 International. 


\title{
La mise en scène de l'écrivain comme sacrifié dans la littérature du premier romantisme allemand : une nouvelle forme de sacrifice?
}

Novalis et la mise en scène de l'écrivain à la lumière des théories de René Girard

\author{
Helene Tessier Amorim
}

1 Girard a beaucoup étudié le roman. Il en tire ses premières idées menant à sa théorie mimétique. Il tisse dès lors un lien particulièrement intéressant entre littérature et anthropologie. L'écriture elle-même n'a cependant pas attiré son attention. Le premier romantisme allemand donne pourtant un exemple de permanence et transmission d'un sacrifice particulier, où la littérature elle-même se met en scène comme sacrifice.

Wackenroder, Tieck, et surtout Novalis nous proposent une mise en scène originale et neuve de l'écrivain au tournant de 1800 . Ces auteurs du premier romantisme allemand cherchent à redéfinir le poète et l'acte de création dans une période marquée à la fois par une crise esthétique et une crise religieuse. De nombreux éléments posent la question d'un rapprochement possible entre théorie mimétique et écriture et on peut se demander en quoi les théories de Girard éclairent le cheminement de pensée du premier romantisme et sa mise en place d'une nouvelle figure de l'écrivain.

Je m'intéresserai ici aux romans et poésies de Novalis notamment, qui mettent en scène l'écrivain et sont le miroir de sa pensée, car comme le résumera si bien Jean-Luc Nancy dans son ouvrage L'Absolu Littéraire, pour les romantiques : "Seul un roman est à même de comporter sa propre réflexion et de comprendre la théorie de son "genre » (ou la loi de son engendrement, c'est la même chose)» (Lacoue-Labarthe, 1978: 275). Il s'agira donc bien d'interroger, à travers ces écrits de prose, une littérature se mettant en scène comme sacrifice, et non le sacrifice dans la littérature. 
René Girard nous montre dans ses ouvrages l'omniprésence du sacrifice. Je voudrai ici davantage en interroger la permanence, autrement dit la transmission. Jean-Luc Nancy insiste sur la transmission et permanence du sacrifice qui ressurgirait sous forme sécularisée dans la littérature, par exemple (Nancy, 1990).

Il faut tout d'abord souligner que Novalis semble transformer son expérience personnelle en exigence existentielle. Il sombre en effet dans un profond désespoir à la mort de sa fiancée Sophia. Ce désespoir constituera le point d'origine d'une réflexion neuve sur l'artiste et son acte de création. Behler, grand connaisseur du romantisme, insiste sur le tournant que marquera cette perte. La mort de Sophia changera profondément la pensée et personnalité du jeune écrivain (Behler, 1992: 58). Dans une phrase restée célèbre, Novalis déclare lui-même, que désormais sa tâche sera de tout rapporter à la mort de sa bien-aimée. Cette mort aura ainsi une influence tout à fait considérable sur son art et sa réflexion poétique (Ibid.).

Cette période est également marquée par une fascination pour le Christ qui n'est pas sans rappeler tout l'intérêt que porte Girard à ce personnage. Au début de l'année 1799, année où il projette également de revenir sur la tombe de Sophia (Ayrault, 1969 : 508), Novalis s'intéresse de près au christianisme. Dans ses Cantiques, poèmes écrits en 1800, le poète parle de son expérience personnelle et évoque sans cesse deux figures principales: le Christ et Sophie, débouchant sur une fusion de la bien-aimée et du Christ. On voit se confondre dans le premier poème son expérience du Christ et de Sophia. De qui parle-til ? L'absence des deux, le sentiment d'abandon, de solitude puis de désespoir, les pleurs liés à leur mort sont mis sur un même plan. Puis, on voit apparaître l'évocation claire du Christ, qu'il évoque comme lumière dans l'obscurité. C'est donc du côté du Christ qu'il faudra chercher, pour comprendre la mort à la fois de ce dernier mais également celle de la bien-aimée. L'étreinte amoureuse et la communion apparaissent comme analogues. Un thème va alors attirer son attention. On sent dans le septième poème, aussi appelé Abendmahlhymne - hymne de la Cène, son intérêt tout particulier pour l'eucharistie et le mystère qu'elle représente. Il s'agit pour Girard également d'un point clé dans sa théorie du sacrifice. Il l'évoque comme tournant. «Bien peu savent » (Novalis, 2014 : 97) nous dit Novalis dès le premier vers comme pour mettre en avant une dimension mystérieuse. De nombreux passages soulignent sa réelle fascination pour l'eucharistie: "celui qui mangera son corps et boira son sang », il emploie également le terme "Cène " associé à «mystère » (apud Dumont, 1969 : 513). Encore une fois le rapprochement de Sophie et du Christ est évident, mais aussi inversé, comme deux figures se répondant dans cette recherche de sens: celui qui a bu sur des lèvres chaudes et aimé l'haleine de la vie comprendra le mystère du pain et du vin. Dans une note, Novalis écrira que le cœur est la clef du monde et de la vie, concluant ensuite que le Christ est le sens de la vie (apud Ayrault, 1969: 515). Mais cette fascination restera un premier temps sans réponse : « Qui peut dire qu'il comprend le sang? » (Ibid. : 511). Novalis se tient face à un mystère, celui de la mort, - mais on perçoit aussi déjà celui de la mort-renaissance - semblant tout aussi indéchiffrable que des hiéroglyphes (métaphore qu'il aime employer). D'ailleurs, il considère que nous n'avons pas compris l'eucharistie et c'est cela qui nous rend « seelenlos» (Kluckhohn, 1977: 159-178), autrement dit sans âme, seul, abandonné. Girard de son côté semble penser la même chose, nous livrant dans son ouvrage le sens et la place à donner au sacrifice du Christ ; ce sur quoi nous reviendrons.

7 Friedrich Schlegel va à ce moment jouer un rôle décisif. Il propose de retourner aux Grecs, aux mythes, à la tragédie qui contiendraient selon lui les réponses. Il serait 
intéressant de rappeler, sans pouvoir nous y attarder longuement, qu'ils façonneront leur pensée à la lecture de la mythologie grecque et romaine ou encore des tragédies d'Euripide et de Sophocle, des pièces donc qui font l'objet de longs développements chez Girard. Schlegel, dans son ouvrage Orphische Vorzeit, évoque Ædipe, Antigone, Achille (Schlegel, 1798 : 21). Dans ce même écrit, il dit toute sa fascination pour les sociétés pratiquant un sacrifice humain ou animal (Schlegel, $1798: 11$ ), puis évoque le passage du sacrifice primitif au don: «les autels des Dieux n'étaient plus tachés de sang, mais on y offrait des gâteaux avec du miel et des fruits, et d'autres sortes de sacrifices similaires " (Schlegel, 1798: 38) - Schlegel utilise le mot Opfer signifiant sacrifice, et que l'on comprend ici dans le sens de don. On y lit une prise de conscience progressive de la permanence du sacrifice. Il écrira dans les premières pages de son ouvrage : « là où il $\mathrm{y} a$ des pratiques, il y a aussi des légendes, et les légendes sont devenues des poèmes au sein de ce peuple » (Schlegel, 1798:5) (Il faut garder à l'esprit que la poésie est aux yeux des romantiques toute forme d'écrits poétiques). Girard souligne dans les textes de l'antiquité grecque notamment les mécanismes sacrificiels qui y sont partout présents, l'origine de la violence, les manifestations du désir mimétique et le rôle du bouc émissaire. C'est peutêtre le plus grand apport des concepts de Girard, car ses réflexions permettent de créer un lien entre les différents intérêts des romantiques et de les ramener à une idée : le sacrifice. La pensée de Girard, en étant le lieu privilégié de la rencontre entre anthropologie et études littéraires, éclaire les paroles de Schlegel dans son ouvrage Orphische Vorzeit : l'étude de la poésie grecque est le chemin le plus court pour découvrir le caractère de la poésie moderne (Behler, 1992: 121); dans la mythologie l'invisible devient visible. Afin de retrouver la clé de la production artistique, il n'y pas de spéculation, il faut chercher la clé dans les textes qui nous parlent tous de la même chose; on pourrait ici entendre parler Girard. La poésie se fonde clairement sur l'anthropologie (Behler, 1992: 70) et des mécanismes pensés comme fondamentaux; les mythes témoignent de ces derniers de manière voilée. Pour soulever en partie le voile, les romantiques se tournent vers la tragédie. Schiller s'est moqué de l'hypothèse de Schlegel d'une harmonie dans les pièces de Sophocle, pourtant, à bien des égards, on voit s'y dessiner le mécanisme dépeint par Girard : « Edipe s'arrache les yeux, Jocaste se pend, tous les deux innocents, la pièce se résout harmonieusement " (apud Behler, 1983 : 345). Schiller n'a pas vu le mouvement qu'y voient les romantiques et sur lequel nous reviendrons. Girard nous dira d'ailleurs que la tragédie a une place clé puisqu'elle fait un pont entre mythes et christianisme; les romantiques y voient un point culminant et remontent en outre à l'origine de la tragédie qui accompagnait les sacrifices primitifs. Selon Girard, les traces de la crise sacrificielle et de sa résolution y seraient ainsi présentes et plus déchiffrables que dans les mythes (Girard, 2010 : 100). Ainsi, la tragédie fournirait « une voie d'accès privilégiée aux grands problèmes de l'ethnologie religieuse » (Girard, 2010: 86). C'est le cheminement que semblent effectivement emprunter Novalis et Schlegel. Tous ces textes suggèrent ainsi, dira Girard, « une présence constante de la crise sacrificielle qu'un seul mécanisme symbolique ne cesse de nous désigner mais de façon voilée » (Girard, 2010: 95). Girard éclaire d'une manière toute particulière le cheminement des premiers romantiques qui se donnent comme tâche principale de percer le secret de la mort, car «la poésie romantique repose tout entière sur un fond historique, bien plus qu'on ne le sait et qu'on ne le croit (Lacoue-Labarthe, 1978 : 326).

8 Le point de convergence que les romantiques tirent de leurs diverses études sera le concept de perte. La fascination pour la perte est révélée par la place centrale qu'elle occupe dans leurs divers textes en prose : perte de l'âge d'or, perte des Dieux ou encore 
point culminant : perte de la femme. Leur travail sur les mythes, la tragédie et la Bible leur révèle une omniprésence de la perte. Un extrait de Henri d'Ofterdingen témoigne de cette révélation et met en lumière cet ouvrage comme réelle mise en scène de l'écrivain, là où Henri et Novalis semblent se confondre : « Tous les hasards semblaient converger et s'unir pour sa formation ", « Tout ce qu'il voyait, tout ce qu'il entendait n'était que pour, semblait-il, lui ôter un nouveau verrou au-dedans de lui-même »; il évoque « la diversité de ses modalités entremêlées et sans cesse changeantes » et enfin « une adorable jeune fille " dont l'évocation de la "tendre bouche » et des "lèvres " rappelle le septième cantique et le rapprochement entre le Christ et la femme aimée (apud Guerne, 1975 : 130). Le désespoir après la mort de Sophie entraina ainsi une réflexion sur la perte qui débouchera sur la conviction de la nécessité de la perte. Girard nous a montré dans ses ouvrages la place centrale ainsi que le rôle déterminant que joue le sacrifice pour la stabilité d'une communauté. Son travail fait apparaître les traces plus ou moins cachées du sacrifice en en révélant l'omniprésence. Le travail des romantiques débouchera sur leur révélation majeure qui réside dans le rapprochement de Dionysos et du Christ qui leur apporte une dernière confirmation; la découverte du dionysiaque est certainement la marque du romantisme allemand, car ni le Klassik ni le Sturm und Drang n'aura vu l'antiquité grecque sous cet angle (Behler, 1983 : 336). Comme le dira Girard, il faut voir derrière les orgies et la fête, la crise mimétique qui les a précédée; Schlegel affirme de son côté qu'il y a un mystère qui les entoure et que c'est là que se trouve la clé. Il ne faudrait pas les considérer comme étant étrangères à nous, comme des dérèglements ou excès nous étant extérieurs (Schlegel, 1798: 22). Les points communs entre les deux personnages, entre autres morts puis ressuscités, sont nombreux. Les romantiques redécouvrent le côté sombre en mettant fin à l'opposition bien-mal, positif-négatif. Ceci marque un tournant dans la pensée de Novalis qui repense alors les concepts de mort et de vie. Le poème intitulé Le chant des morts contient deux vers très frappants : «La mort est le but suprême de la vie " (Kluckhohn, 1977: 350) et en fin de poème, "apprend à saisir le sens de la mort » (Kluckhohn, 1977 : 354). L'idée que la mort est le sens de la vie donne à comprendre que les deux termes ne sont plus pensés en opposition, mais davantage dans une relation dynamique fondée sur un principe de nécessité. Elle permet d'installer la dynamique perte-gain au centre de toutes leurs réflexions. Cette dynamique peut être vue comme une sorte de dialectique avant l'heure. Novalis et Schlegel emploient les termes de Selbstvernichtung-Selbstschöpfung (Behler, 1992: 162); il s'agit d'un mouvement ininterrompu d'autoproduction et autodestruction (Behler, $1983: 340$ ) et qui est à la base de tout. Toute leur pensée est basée sur le mode de la dynamique des contraires (Lacoue-Labarthe, 1978). C'est exactement ce qu'ils voient chez Sophocle et que Schiller n'a pas compris, ce mouvement de nécessité puis d'harmonie qui sans cesse se répète. Quand Jean-Luc Nancy creuse la théorie du premier romantisme, on s'aperçoit très vite de la place centrale de la dynamique (Ibid.); tandis que Kluckhohn, dans son anthologie, nous dit dans la préface qu'il y a finalement une chose - cette idée de dynamique - à la base de tout dans la pensée du romantisme (Kluckhohn, 1977: 23). Girard nous permet de remonter et comprendre la mise en place d'une telle conception de l'art et de l'acte d'écriture.

9 Cette révélation est donc le terme d'un processus de pensée parti de la mort de Sophie et des réflexions sur l'eucharistie, éclairées par les mythes et la tragédie. Pour arriver au rapprochement entre art et sacrifice chez les premiers romantiques, il faut ainsi passer par le mouvement même de la réflexion de Novalis qui trouve son expression dans Les 
hymnes à la nuit. Il y résume son cheminement entre les hymnes 3 et 5 . Le poète passe du désespoir et de l'incompréhension de sa situation dans l'hymne 3 :

Un jour que je versais des larmes douloureuses, que mon espoir, bientôt évanoui dans la souffrance, s'en allait en ruisselant, et que je me tenais seul auprès du tertre sec, dont l'espace étroit et sombre abritait la forme de ma vie, solitaire comme aucun solitaire ne l'a jamais été, acculé et poussé par une angoisse indicible, sans force, j'étais à peine l'idée de détresse (...) (apud Dumont, 2014 : 7)

Puis l'hymne 4 témoigne de la révélation :

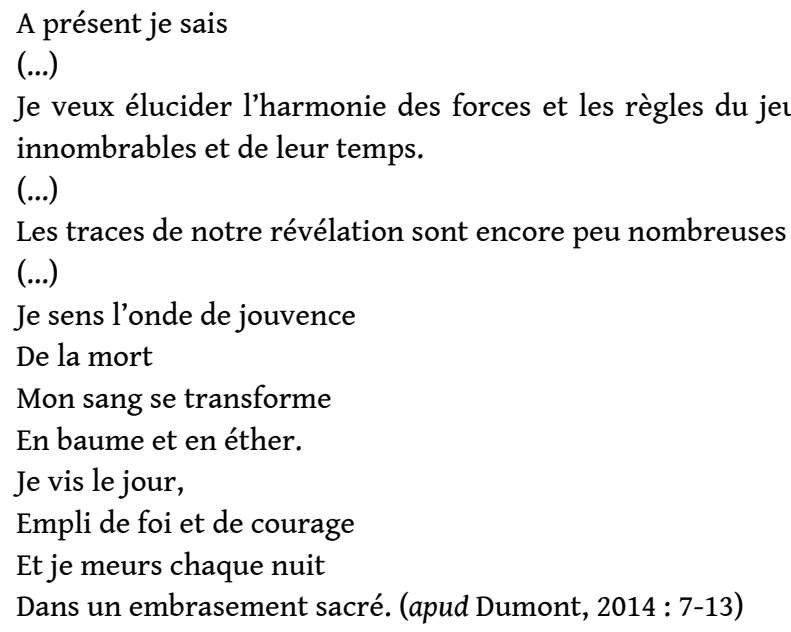

11 Le cinquième hymne est enfin considéré comme illustrant la théorie de l'écrivain. En son centre, la mort et la résurrection; il reprend les références de l'antiquité au Christ, faisant apparaître une cohérence et une unité.

Dans la mort se révélait la vie éternelle,

Tu es la mort et tu nous guéris pourtant.

(...)

Quelques jours seulement, un voile profond demeura suspendu sur la mer mugissante, sur la terre ébranlée

(...)

Le mystère fut descellé

(...)

Désormais, celui qui croit et aime

Ne pleure plus auprès d'aucun tombeau.

(...)

La nuit l'embrase et l'inspire. (apud Dumont, 2014 : 21-25)

12 Le IV ${ }^{e}$ cantique évoque également un univers en ruine, la souffrance et la peine du poète face à la tombe, puis, en dernière strophe, l'illumination intérieure de Novalis avec sa singularité et son mystère, se terminant encore une fois sur une référence au sacrifice par l'image de la plaie (Ayrault, 1969: 507). Il interprète alors la mort de Sophie comme « himmlischer Zufall » - hasard divin, «Schlüssel zu allem » - clé de tout, donc nécessaire (Kluckhohn, $1977:$ 15).

13 L'eucharistie est un point clé, c'est ce que pense également Girard, qui permet ainsi d'apporter un éclairage inédit sur la réflexion de Novalis. Dans la Chrétienté ou l'Europe, Novalis souligne que la Réforme oublia le principal dans le processus et ne proposa pas d' ersatz suffisant (Kluckhohn, 1968 : 511), rappelant ici les disputes au sujet de l'eucharistie ; la simple commémoration proposée par le protestantisme est sèche, finira-t-il par dire. On retrouve l'idée évoquée par Girard de la puissance du processus sacrificiel réactualisé et répété dans l'eucharistie qui fait revivre le sacrifice du Christ. Le catholicisme garde le 
secret de la dynamique sacrificielle pour les Romantiques. À la fin du texte La Chrétienté ou l'Europe, Novalis souligne sa qualité de "Zeugungselement » (Kluckhohn, 1968 : 523), c'est à dire de production. Or, à travers tout ce cheminement, il recherche la clé de l'écriture. Le passage et la transmission de mécanismes fondamentaux, un temps au moins religieux, sous forme sécularisée se fait ici jour.

Novalis et son génie du christianisme, comme le dira Ayrault ; cela rapproche sans aucun doute Novalis de Girard. Quand Novalis dit que «Le repas en commun est un acte symbolique d'union... (...) le mystérieux moyen d'une transfiguration et d'une divinisation ici-bas (...)» (Ayrault, 1969: 512), on ne peut s'empêcher de penser à Girard et à la stabilité retrouvée après une situation de crise, au rôle du bouc émissaire et au caractère inconscient nécessaire au fonctionnement du mécanisme. Mais les romantiques ne semblent pas voir jusque-là; ils observent avec fascination la perte sans en percer totalement le secret. On se situe ici à un point de paradoxe, qui n'est pas sans être évoqué par Girard lui-même. Certes, le sacrifice du Christ dévoile le mécanisme, cependant les romantiques ne remontent pas comme Girard jusqu'au désir mimétique, à la violence réciproque; ils voient le sacrifice, le bouc émissaire et la dynamique perte-gain ; ils y voient finalement la clé de toute création, de toute production. En s'appuyant sur la pensée de Schelling, l'intuition est à la base de leur pensée qui trouvera finalement sa confirmation à travers leurs recherches ; ils sont persuadés qu'il est impossible à l'homme de tout comprendre. Il reste une part de mystère à laquelle nous n'avons pas accès. Ainsi, leur conviction que toute chose est marquée par une dynamique de perte et de gain montre une reprise du sacrifice à la fois consciente, puisque correspondant à une nécessité personnelle et s'appuyant sur de nombreuses recherches, et inconsciente, car le mystère du mécanisme du sacrifice reste mystère lorsque le voile sera soulevé. Dans cette reprise du sacrifice, c'est-à-dire de la dynamique, découlant et confirmée par leurs multiples références - ou comme dirait Girard les multiples traces qu'ils découvrent - un exemple en particulier fera figure de modèle : le sacrifice du Christ. Il est la clé du monde, disait Novalis, et on croirait entendre Girard, car il s'agit d'un auto-sacrifice qui témoigne d'une dimension nouvelle et d'un changement de perspective permettant ainsi une reprise chez l'artiste.

Le Christ ne met pas fin au sacrifice, mais nous en propose une nouvelle forme, mimésis de l'ancien mais cherchant à en garder tout l'effet; il témoigne et ouvre le passage vers l'individualisation accompagnant l'intériorisation et la spiritualisation du sacrifice, car c'est bien le dévoilement partiel qu'il contient qui engendre la réflexion des romantiques. Comment les romantiques ne pourraient-ils pas voir désormais dans l'eucharistie une dynamique perte-gain qui sans cesse se répète, chaque dimanche. L'exemple de Jonas et la baleine développé par Girard met en lumière l'écriture et la mise en scène du poète chez les romantiques comme une reprise sécularisée de vieux mythes sacrificiels, car c'est justement cette histoire tirée de l'Ancien Testament que reprendra Novalis dans un de ses romans (Kluckhohn, 1977: 211). Henri d'Ofterdingen, parti sur les routes, rencontre diverses personnes qui lui racontent des histoires, dont celle d'un poète d'un temps reculé, qui voulait aller découvrir des terres inconnues. Il trouve un bateau contre une rémunération importante. Mais une fois parti, la richesse du poète attise les désirs de l'équipage. Ils choisissent de le tuer, car craignent d'être dénoncés malgré la promesse du poète. Ce dernier promet ensuite de se jeter lui-même à la mer s'ils lui accordent une dernière volonté. Il chante et les matelots décident de se boucher les oreilles pour ne pas changer d'avis; on pourrait ici réfléchir à la portée anti-mimétique de l'écriture, 
introduite par Novalis, car ce passage n'existe pas dans le texte d'origine. Le poète sautera donc, mais peu après, la crise engendrée par un désir mimétique ne trouve pas d'exutoire et la violence de tous contre tous les amènera à s'entretuer. Girard avait repris le texte biblique dont s'inspire clairement Novalis (Girard, 1982: 174), afin de montrer que l'Ancien Testament regorge de personnages qu'il faut dévoiler comme victime, bouc émissaire ; en effet Jonas est la victime émissaire que les marins jettent par-dessus bord au cours d'une tempête. Encore une fois, les romantiques y voient la perte et le gain. Reprendre de manière aussi évidente et flagrante un texte biblique, souligne en outre clairement l'association que Novalis fait entre les boucs émissaires de la Bible et le poète, et met en scène le poète comme sacrifié voire auto-sacrifié. Il fait apparaître dans la réécriture d'autres aspects de la crise mimétique et surtout change le sacrifice subi par le prophète en un sacrifice choisi par le poète sur lequel nous reviendrons. Dans la deuxième partie de l'ouvrage non écrite, Novalis rédige une note qui cette fois relie sacrifice primitif et poète : «Dissolution d'un poète dans son chant - il devra être sacrifié parmi des peuples sauvages » (apud Kluckhohn, $1977: 337$ ). On trouve dans ces exemples la double référence à des sacrifices tirés à la fois de l'ethnologie et de la Bible.

Ces passages révèlent la littérature comme étant une reprise sécularisée de vieux mythes sacrificiels et mettent en lumière la transmission et transformation du sacrifice jusqu'au romantisme.

Le véritable sacrifice semble en effet dès lors être celui de l'artiste et devenir le moteur de la création. La théorie mimétique de Girard trouve cependant peut-être ici son prolongement dans des penseurs tels que Nancy ou Blanchot, car cette forme de sacrifice - l'autosacrifice du Christ - trouve sa limite, soulignera Nancy (1990: 86); si le sacrifice permet l'écriture, il ne peut être un suicide (Blanchot, 1955 : 138), il répond à une nouvelle exigence nous dit Blanchot dans divers textes (Blanchot, 1983). Il prend en effet une forme nouvelle, il n'est plus l'autosacrifice du Christ, même si ce dernier en propose le modèle. Par ailleurs, Orphée offre un dépassement, , face à l'impasse d'un sacrifice physique, au moment où ces écrivains doivent trouver dans la reprise du sacrifice une forme adéquate entre simulacre et néant, permettant la réappropriation de la dynamique du sacrifice, mais également au bout l'écriture. Le sacrifice ne peut pas se terminer sur une mort physique, mais doit plutôt permettre l'ouverture de l'espace d'écriture lors d'un instant d'extase de l'artiste, comme suspendu au bord du gouffre. L'intérêt pour Orphée n'est pas nouveau, car des poèmes de jeunesse de Novalis lui sont consacrés, mais l'intérêt pour ce personnage ressurgit dans ces années 1799 sous un tout nouveau jour, en lien cette fois avec leurs découvertes sur les mythes, tragédies et en prolongement de l'exemple du Christ. Le personnage Henri d'Ofterdingen ne deviendra artiste qu'à la mort de sa bien-aimée et quand il exprimera le choix de s'y consacrer. Soulignons également que dans l'histoire de Jonas reprise par Novalis, Jonas ne meurt pas, car il est sauvé par une baleine. Novalis nous dit dans sa version que le poète saute dans l'abîme sombre, il ne dit pas la mer; on peut ainsi le comprendre métaphoriquement. Cela rappelle la redécouverte du côté sombre, associé comme partie indispensable de la dynamique et perdant son côté habituellement négatif ou angoissant. En outre, le saut du poète, droit et tête haute, montre qu'il s'agit d'un autosacrifice, d'un choix qui prendra la forme du don de soi, de la souffrance choisie. D'abord désigné par les autres, il n'en ressaisit pas moins le moment de son sacrifice, le transformant ainsi en autosacrifice. Enfin, le chant et la musique qu'il produit sur le bateau sont d'une particulière beauté et semblent animer nature et animaux qui l'entourent. Ceci n'est pas sans rappeler Orphée. Dans la deuxième 
partie non écrite de l'ouvrage Henri d'Ofterdingen, Novalis évoque une partie qu'il projette d'écrire sur une légende d'un poète qui partirait dans les ténèbres chercher une personne (Kluckhohn, 1977 : 337). Schlegel enfin dans son ouvrage Orphische Vorzeit s'intéresse de très près au personnage d'Orphée, rappelant que les hellènes avaient leur propre poésie "à la tête de laquelle on trouvait la légende d'Orphée, le père de la Poésie » (1798: 5). Il précise ensuite en s'appuyant toujours sur Orphée qu'il faut prendre de la distance avec la simple plainte suite à la mort de la bien-aimée afin d'arriver au chant (Schlegel, 1798 : 29); c'est bien ce mouvement que nous avons vu dans les Cantiques et les Hymnes à la nuit. Par ces multiples références à Orphée, la souffrance et le renoncement apparaissent comme la nouvelle expression du sacrifice nécessaire du poète qui s'inscrit dans une dynamique essentielle de perte et de gain qui semble être à la base de toute production et deviendra ainsi également la condition ou plutôt la clé de la production littéraire.

Ces multiples références - aux mythes, à la tragédie, à l'Ancien Testament, au Christ - qui font apparaitre le romantisme comme un ensemble de réflexions complexes et hétéroclites - sont éclairées par les théories de René Girard qui nous offre un appareil conceptuel qui semble tout à fait se prêter à l'analyse du cheminement de pensée de Novalis. La conception de ce dernier de l'acte d'écriture et sa mise en scène de l'écrivain, dont nous avons eu un aperçu à travers différents extraits, fait apparaître la littérature comme une réécriture de vieux mythes sacrificiels, transmis à travers le temps et transformés lorsqu'ils réapparaissent sous forme sécularisée dans la littérature. Les Romantiques nous offrent l'exemple d'une reprise à la fois consciente et inconsciente du sacrifice et l'installent comme point clé de l'écriture. Derrière le sacrifice, se cache le secret de toute production et derrière l'eucharistie, c'est-à-dire l'autosacrifice du Christ, celui de toute autoproduction. Si Girard apporte un éclairage sur leur cheminement de pensée et permet de mettre en lumière le point commun à toutes leurs recherches, il faudra cependant se tourner vers d'autres penseurs tels que Blanchot ou Nancy pour appréhender la forme que prendra par la suite le sacrifice chez l'écrivain romantique.

\section{BIBLIOGRAPHIE}

AYRAULT, Roger (1969). La Genèse du romantisme allemand. Tome 3 : 1797-1804 (I) Baader ; Brentano ; Hülsen ; Novalis ; Ritter ; Schelling ; Steffens ; les Schlegel : Auguste Wilhelm, Caroline, Dorothée, Friedrich ; Schleiermacher ; Tieck; Wilhelm Heinrich Wackenroder. Paris : Aubier - Éditions Montaigne.

BEHLER, Ernst (1983). Die Auffassung des Dionysischen durch die Brüder Schlegel und Friedrich Nietzsche, in Ernst Behler, Mazzino Montinari, Wolfgang Müller-Lauter (éds.). Nietzsche-Studien Bd. 12.

Berlin : De Gruyter, pp. 335-354.

BEHLER, Ernst (1992). Frühromantik. Berlin : De Gruyter.

BLANCHOT, Maurice (1983). La Communauté inavouable. Paris : Les éditions de minuit.

BLANCHOT, Maurice (1955). L’Espace littéraire. Paris : Gallimard. 
DUMONT, Augustin (2014). Novalis. Hymnes à la nuit, chants spirituels, disciples à Saïs. Paris : Les belles lettres.

GIRARD, René (2010). La Violence et le sacré. Paris : Pluriel.

GIRARD, René (1982). Le Bouc émissaire. Paris: Grasset.

GUERNE, Armel (1975). Novalis. Henri d'Ofterdingen. Paris : Gallimard.

KLUCKHоHN, Paul, SAMUEL, Richard (1977). Novalis Schriften. Band 1. Darmstadt : Wissenschaftliche Buchgesellschaft.

KLUCKHOHN, Paul, SAMUEL, Richard (1968). Novalis Schriften. Band 3. Darmstadt : Wissenschaftliche Buchgesellschaft.

LACOUE-LABARTHE, Philippe, NANCY, Jean-Luc (1978). L'Absolu littéraire. Théorie de la littérature du romantisme allemand. Paris : Seuil.

NANCY, Jean-Luc (1990). Une Pensée finie. Paris : Galilée.

SCHLEGEL, Friedrich (1798). Geschichte der Poesie der Griechen und Römer. Berlin : Johann Friedrich Unger.

\section{RÉSUMÉS}

Le premier romantisme allemand donne l'exemple de la permanence et transmission du sacrifice à la fois consciente et toujours inconsciente, malgré la révélation du mécanisme par le christ, lorsque Wackenroder, Tieck et surtout Novalis nous proposent une mise en scène originale et neuve de l'écrivain. La redéfinition du poète et de l'acte de création autour de 1800 permet un rapprochement possible entre théorie mimétique et écriture. Girard semble offrir un éclairage nouveau sur le cheminement des premiers romantiques allemands. Dans leurs romans autoréflexifs, le sacrifice prend une forme nouvelle, au moment où ces écrivains doivent trouver dans la reprise du sacrifice une forme adéquate entre simulacre et néant, permettant la réappropriation de la dynamique du sacrifice, mais également, au bout, l'écriture, la production littéraire.

Jena Romanticism can be used as an example to illustrate the conscious and unconscious permanency and transmission of sacrifice, and this despite Christ's revelation of the mechanism, as Wackenroder, Tieck and Novalis offer us a new and original staging of the writer. The redefinition of the poet and of the act of creation around 1800 allows a reconciliation between mimetic theory and writing. In their self-reflexive novels, the sacrifice takes a new form, when these writers must find in the resumption of sacrifice an adequate form between travesty and nothingness, allowing the reappropriation of the dynamics of sacrifice, but also, at the end, writing, literary production.

\section{INDEX}

Keywords : mimetic theory, jena romanticism, literature, sacrifice, writing

Mots-clés : théorie mimétique, premier romantisme allemand, littérature, sacrifice, écriture 
AUTEUR

HELENE TESSIER AMORIM

Université de Rouen

helene.tessier1[at]univ-rouen.fr 